\title{
A Case of Intractable Suspected Perilymph Fistula with Severe Depression
}

\author{
Fumiyuki Goto ${ }^{1,2} \bowtie$, Naoki Oishi ${ }^{2}$, Tomoko Tsutsumi ${ }^{3}$, and Kaoru Ogawa ${ }^{2}$ \\ ${ }^{1}$ Department of Otorhinolaryngology, National Hospital Organization Tokyo Medical Center, Tokyo, Japan \\ 2Department of Otolaryngology, Head and Neck Surgery, Keio University School of Medicine, Tokyo, Japan \\ ${ }^{3}$ Department of Otolaryngology, Hino Municipal Hospital, Tokyo, Japan
}

\begin{abstract}
A 68-year-old woman presented dizziness whenever she put her finger into the right ear and also complained of water-streaming tinnitus, which indicated she would have been suffering from perilymph fistula. An exploratory tympanotomy was conducted. Leakage of perilymph from the round window was suspected, although the cochlin-tomoprotein (CTP) results were negative. After the procedure, the patient's finger-induced dizziness, tinnitus, and vertigo spells disappeared completely. However, her dizzy symptom did not improve. The patient also complained of general fatigue, weight loss, and insomnia, which led us to suspect comorbid depression. Antidepressants and vestibular rehabilitation treatment resulted in a significant improvement in her dizziness. Although it is not apparent whether the patient had a perilymph fistula, this case demonstrates the importance of evaluating not only physical symptoms but also psychological comorbidity, especially when the physical symptoms are intractable despite treatment.

Psychiatry Investig 2014;11(4):499-501
\end{abstract}

Key Words Depression, Perilymph fistula, Vestibular rehabilitation.

\section{INTRODUCTION}

Somatic symptoms that cannot be explained by a physical examination are termed "medically unexplained symptoms" (MUS). ${ }^{1,2}$ Tinnitus and dizziness are examples of MUS. Individuals with MUS often visit an otolaryngology clinic to seek treatment. Depression has been implicated as a major comorbidity in patients with chronic otolaryngologic MUS. ${ }^{1} \mathrm{Al}-$ though the prevalence of depression among all otolaryngological patients has been reported to range from $10-26 \%,{ }^{3,4}$ the exact prevalence of depression among patients with otolaryngological MUS is uncertain.

We previously reported that depression may be difficult to diagnose in otolaryngology patients with MUS. ${ }^{5}$ In fact, it has been reported that $73.5 \%$ of patients with major depression complain of somatic rather than psychiatric symptom; ${ }^{6}$ this

Received: June 13, 2013 Revised: September 18, 2013

Accepted: September 30, 2013 Available online: October 20, 2014

$\triangle$ Correspondence: Fumiyuki Goto, MD, PhD

Department of Otorhinolaryngology National Hospital Organization Tokyo Medical Center, 2-5-1 Higashigaoka Meguro, Tokyo 152-0021, Japan

Tel: +81-3-3411-0111, Fax: +81-3-3412-9811

E-mail: amifumi@bc5.so-net.ne.jp

(a) This is an Open Access article distributed under the terms of the Creative Commons Attribution Non-Commercial License (http://creativecommons.org/licenses/by$\mathrm{nc} / 3.0$ ) which permits unrestricted non-commercial use, distribution, and reproduction in any medium, provided the original work is properly cited. phenomenon is known as "masked depression." Here, we report a case of suspected perilymph fistula with severe depression, which was successfully treated with antidepressants and vestibular rehabilitation.

\section{CASE}

A 68-year-old woman presented with frequent vertigo spells and long-standing dizziness accompanied by right-sided tinnitus after acute sensorineural hearing loss in the right ear several months before. When the patient was 24 years old, her left ear was surgically treated for chronic otitis media. Her pure tone audiometry (PTA) score as measured on her first visit revealed bilateral mixed hearing loss (Figure 1). An otoscopic examination of her left ear revealed the finding of postoperative tympanic membrane without any evidence of active inflammation and the enlargement of external auditory canal. The right ear was normal in appearance. However, when an otoscope was inserted in her right ear, the patient immediately complained of dizziness. She then admitted to feeling dizzy whenever she put her finger in her right ear. It was assumed that insertion of the otoscope exerted positive pressure to the external auditory canal and thereby provoked subjective dizziness. A charge-coupled device (CCD) camera was used to record all subsequent observations. The application of negative 


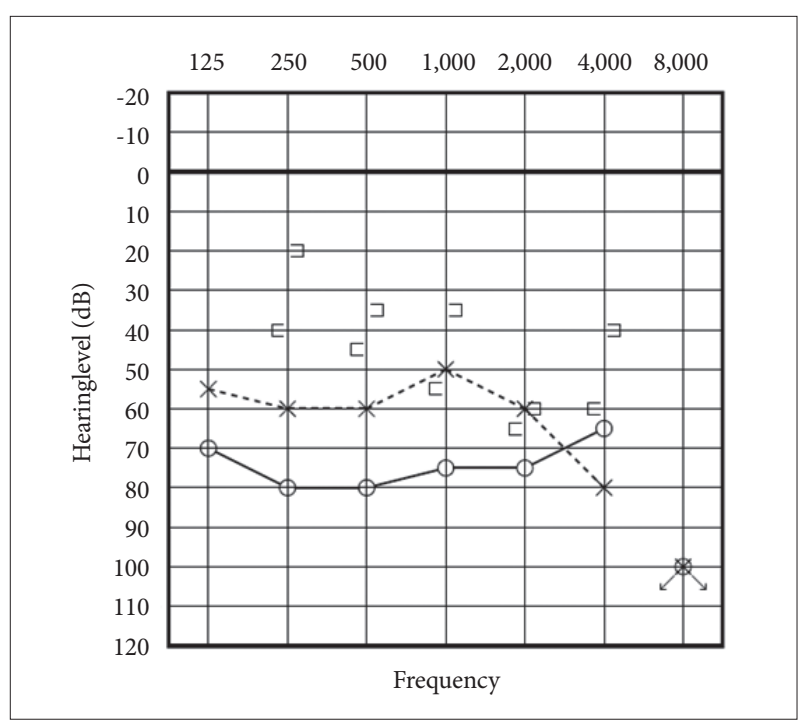

Figure 1. Pure tone audiometry (PTA) upon first presentation to our hospital. The PTA revealed that the patient had bilateral mixed hearing loss. The patient had undergone a left tympanoplasty 42 years previously. She reported a 2-month history of acute sensorineural hearing loss in the right ear.

air pressure to the patient's right external auditory canal induced nystagmus toward the right side as well as a dizzy sensation. The patient also felt dizzy when changing her body position, but this sensation was not associated with apparent nystagmus. ${ }^{7}$ The patient also complained of tinnitus, which she characterized as similar to the sound of water streaming. The caloric test with ENG indicated canal paralysis of $35 \%$ in right ear with 20 degree water irrigation. Temporal bone CT did not reveal any findings of pneumolabyrinth or aerolabyrinth. There were no traumatic events to cause perilymph fistula, e.g., excessive pressure changes to the inner ear such as in deep-water diving, head trauma, or an extremely loud noise, etc. From these clinical findings we could not completely rule out the possibility of underlying idiopathic perilymph fistula.

An exploratory tympanotomy was conducted under general anesthesia. During the procedure, the leakage of perilymph was suspected in the round window under the microscope. However, in fact, the leakage of perilymph fluid was not confirmed by an increase of intracranial pressure by postural change (head-down position) or an increase of chest pressure. The tympanic space was irrigated with $0.1 \mathrm{cc}$ of saline. The irrigation solution was collected in order to measure the level of cochlin-tomoprotein (CTP)-a common perilymph marker. ${ }^{8}$ The oval and round windows were sealed to the temporal fascia with fibrin glue.

After the procedure, the patient's tinnitus, finger-induced vertigo, and nystagmus resolved completely. This marked improvement of symptoms suggested that her complaints had stemmed from a perilymph fistula. However, a definitive diagnosis of perilymph fistula was not possible without positive

\section{CTP results.}

Although the surgery seemed to relieve most of the patient's symptoms, she still experienced her constant dizziness, which prevented her full engagement in social activities. In addition, the patient did not regain any hearing in her right ear. She complained of general fatigue, weight loss, and insomnia, which led us to suspect comorbid depression. The clinical evaluation was conducted using self-administered questionnaires. The dizziness handicap inventory (DHI) $)^{9}$ score was 94 (physical, 24; emotional, 34; functional, 36), implying that she was suffering from severe dizziness. The hospital anxiety and depression scale (HADS $)^{10}$ score was 12 for anxiety and 15 for depression, suggesting that she was in a depressive state as well as a state of high anxiety. The patient's postural sway was recorded using static posturography. ${ }^{11}$ The total length of body sway over a period of $60 \mathrm{~s}$ was $166.08 \mathrm{~cm}$ with eyes open and $238.06 \mathrm{~cm}$ with eyes closed. The area of body sway over the same duration was $7.63 \mathrm{~cm}^{2}$ with eyes open and $9.86 \mathrm{~cm}^{2}$ with eyes closed.

On the basis of the questionnaire results, the treatment for depressive mood was considered to be an important issue before introducing vestibular rehabilitation to resolve a vestibular symptom of this patient. The antidepressant (miltazapiene, 7.5 $\mathrm{mg} /$ day) treatment was prescribed. Unfortunately, the patient was forced to stop the treatment owing to an adverse reaction to the medication. Substitute antidepressants (sertraline, 25 $\mathrm{mg}$ and sulpiride, $50 \mathrm{mg} /$ day) were prescribed. This course of treatment was free of side effects and effective in addressing the patient's symptoms. Two weeks after starting this regimen, the patient reported an improvement in her clinical symptoms. With the aid of this pharmacotherapy, a program of vestibular rehabilitation was initiated to accelerate the patient's recovery. Four weeks later, the patient achieved a DHI score of 24 (physical, 8; emotional, 8; functional, 8)and HADS scores of 5 and 6, respectively. Her static posturography results improved as well: length of body sway for $60 \mathrm{~s}, 108.59 \mathrm{~cm}$ with eyes open and $148.56 \mathrm{~cm}$ with eyes closed; and area of body sway for $60 \mathrm{~s}$, $8.09 \mathrm{~cm}^{2}$ with eyes open and $8.14 \mathrm{~cm}^{2}$ with eyes closed.

\section{DISCUSSION}

Patients with unrecognized depression who suffer from various somatic symptoms often consult with their physicians instead of visiting a psychiatrist, which results in the increased consumption of health care resources. ${ }^{12}$ Depressive disorders are substantially under-recognized in primary care clinics including otolaryngology and general hospital settings. ${ }^{13}$

It is truly difficult to treat somatic disorders that present in association with psychiatric problems such as depression. In this case, the suspected perilymph fistula and comorbid de- 
pression rendered an accurate assessment difficult.

The patient's previous clinical history suggested the possibility of a perilymph fistula, ${ }^{7}$ which was further supported by the marked postoperative resolution of most of her symptoms. CTP is a specific diagnosis marker for perilymph fistula; however, negative CTP results prevented a definitive diagnosis. It remains possible that the resolution of symptoms observed after the exploratory tympanotomy was a variant of the placebo effect.

The persistence of dizziness after the surgery suggested a comorbid condition. Weight loss, general fatigue, and insomnia are common symptoms of depression. The HADS results supported our clinical impression. Furthermore, the patient's mental health status prevented her from participating in vestibular rehabilitation. ${ }^{14}$ The prescribed course of treatment improved the patient's mood and allowed her to smoothly and effectively complete a course of vestibular rehabilitation. Interestingly, DHI scores and measures of psychological distress are closely related, ${ }^{15}$ which suggests a potential synergistic effect.

In conclusion, we report that it is important to evaluate not only physical symptoms but also possible psychological comorbidity, especially when the physical symptoms do not resolve even after relevant intervention and treatment.

\section{Acknowledgments}

This study is in part supported by grant from Magnetic Health Science Foundation 2014.

\section{REFERENCES}

1. Ballas CA, Staab JP. Medically unexplained physical symptoms: toward an alternative paradigm for diagnosis and treatment. CNS Spectr 2003;8(12 Suppl 3):20-26.
2. LaFrance WC, Jr. Somatoform disorders. Semin Neurol 2009;29:234246.

3. Bhattacharyya N, Wasan A. Do anxiety and depression confound symptom reporting and diagnostic accuracy in chronic rhinosinusitis? Ann Otol Rhinol Laryngol 2008;117:18-23.

4. Chandra RK, Epstein VA, Fishman AJ. Prevalence of depression and antidepressant use in an otolaryngology patient population. Otolaryngol Head Neck Surg 2009;141:136-138.

5. Goto F, Tsutsumi T, Oishi N, Mimura M. Hidden depression in otolaryngology patients with medically unexplained symptoms. Gen Hosp Psychiatry 2012;34:206-208.

6. Sugahara H, Akamine M, Kondo T, Fujisawa K, Yoshimasu K, Tokunaga $\mathrm{S}$, et al. Somatic symptoms most often associated with depression in an urban hospital medical setting in Japan. Psychiatry Res 2004;128: 305-311.

7. Goto F, Ogawa K, Kunihiro T, Kurashima K, Kobayashi H, Kanzaki J. Perilymph fistula--45 case analysis. Auris Nasus Larynx 2001;28:29-33.

8. Ikezono T, Shindo S, Sekiguchi S, Hanprasertpong C, Li L, Pawankar $\mathrm{R}$, et al. Cochlin-tomoprotein: a novel perilymph-specific protein and a potential marker for the diagnosis of perilymphatic fistula. Audiol Neurootol 2009;14:338-344.

9. Jacobson GP, Newman CW. The development of the Dizziness Handicap Inventory. Arch Otolaryngol Head Neck Surg 1990;116:424-427.

10. Zigmond AS, Snaith RP. The hospital anxiety and depression scale. Acta Psychiatr Scand 1983;67:361-370.

11. Di Fabio RP. Sensitivity and specificity of platform posturography for identifying patients with vestibular dysfunction. Phys Ther 1995;75: 290-305.

12. Simon GE, Chisholm D, Treglia M, Bushnell D; LIDO Group. Course of depression, health services costs, and work productivity in an international primary care study. Gen Hosp Psychiatry 2002;24:328-335.

13. Gilbody S, Richards D, Brealey S, Hewitt C. Screening for depression in medical settings with the Patient Health Questionnaire (PHQ): a diagnostic meta-analysis. J Gen Intern Med 2007;22:1596-1602.

14. Cohen H. Vestibular rehabilitation improves daily life function. Am J Occup Ther 1994;48:919-925.

15. Hong SM, Lee HJ, Lee B, Park SK, Hong SK, Park IS, et al. Influence of Vestibular Disease on Psychological Distress: A Multicenter Study. Otolaryngol Head Neck Surg 2013;148:810-814. 